\title{
A behavioral view of Nevanlinna-Pick interpolation
}

\author{
I. Pendharkar*, H. K. Pillai* and P. Rapisarda ${ }^{\dagger}$
}

\begin{abstract}
The classical Nevanlinna-Pick (NP) interpolation problem is about finding a rational function that satisfies given interpolation conditions, along with a norm condition. In this paper we address the NP problem using concepts from behavioral systems theory and quadratic differential forms (QDFs). The NP problem is solved using a certain "dualization of data". We address system theoretic motivations for this dualization and the advantages gained in this process. Finally, we address the problem of constructing interpolating functions that satisfy a "frequency dependent" norm condition.
\end{abstract}

\section{INTRODUCTION}

A class of interpolation problems in Hardy spaces consists of computing an analytic function that satisfies some interpolation conditions, along with a norm constraint. The Nevanlinna-Pick (NP) interpolation problem is one of the most important interpolation problems in this class. The NP interpolation problem has found numerous applications in model approximation, robust stabilization, the model matching problem in $H_{\infty}$ control, circuit theory, among others. We now give a brief statement of the classical NP problem:

Given $n$ pairs of complex numbers $\left(\lambda_{i}, b_{i}\right)$ with $\lambda_{i} \in \mathbb{C}^{+}$, the open right half complex plane, and $\left|b_{i}\right|<1, i=1,2 \ldots n$, compute a scalar rational function $G(s)$ such that

1) $G\left(\lambda_{i}\right)=b_{i}, i=1,2 \ldots n$

2) $G(s)$ is analytic in $\mathbb{C}^{+}$.

3) $\sup _{\omega \in \mathbb{R}}|G(i \omega)|<1$

Several variants of the above problem have been studied with various assumptions on the data, [2]. The scalar and matrix versions, the tangential NP problem with (simple) multiplicities, the two sided Nudelman problem, the Subspace Nevanlinna Pick Interpolation (SNIP) [7] are some variants and generalizations of the classical NP problem. Basic to all these variants of the NP problem is an assumption of a "frequency independent norm" that the interpolating (rational) function must satisfy. In other words, it is assumed that the norm inequality satisfied by the interpolating function is the same at all frequencies.

In this paper, we re-examine various aspects of the NP interpolation problem in the behavioral theoretic framework [5]. We show that concepts in behavioral theory can be conveniently married to the concepts behind NP-like problems to yield generalizations in several directions. We show that the classical NP interpolation problem can be re-cast into a problem of dissipative systems, on which considerable work

\footnotetext{
*Department of Electrical Engineering, Indian Institute of Technology Bombay Powai, Mumbai - 400 076, India.

$\dagger$ School of Electronics and Computer Science, University of Southampton, United Kingdom.
}

has been reported in behavioral systems theory. In such a setting, the aspects of analyticity and the norm conditions on the interpolant can be examined separately. This formulation also allows us to consider a generalized interpolation problem wherein the required interpolant satisfies a frequencyweighted norm condition.

\section{BACKGROUND AND PRELIMINARY RESULTS}

We assume that the reader is familiar with the basic philosophy of the behavioral approach to systems theory. However, for the sake of completeness, a brief introduction is given below. A "behavior" is, loosely speaking, a set of trajectories in some function space. This set is defined by certain laws. A behavior is called linear if these laws are linear. A linear behavior is called controllable if any trajectory in the behavior can be "steered" to every other trajectory in the behavior (see [5] for the exact definition). A behavior is controllable if and only if it admits a "image representation". A behavior is called "autonomous" if it is finite dimensional. Autonomy is the worst-possible kind of uncontrollability.

\section{A. Models for data}

By "data" we mean a finite set of measurements of a phenomenon. A model $M$ for the data is a subset of the ambient space containing the data. $M$ is often chosen from a "model class" $\mathcal{M}$ which presumes certain features about the model like linearity, time invariance etc. Clearly, by this definition the data admit several models. However, it is possible to order these models according to their prohibitive power: given the data, a model class $\mathcal{M}$, and two models $M_{1}$ and $M_{2}$ for the data from the class $\mathcal{M}, M_{1}$ is said to be more powerful than $M_{2}$ if $M_{1} \subset M_{2}$. A model $M^{*}$ from a model class $\mathcal{M}$ is said to be a Most Powerful Unfalsified Model (MPUM) for the given data if it contains the data and if every other model $M$ from $\mathcal{M}$ for the data satisfies $M^{*} \subseteq M$.

In the sequel, we assume that the data is given as

$$
\mathcal{D}=\left\{\mathcal{V}_{i} \exp _{\lambda_{i} t}, i=1,2 \ldots, n, c_{i} \in \mathbb{C}^{q}, \lambda_{i} \in \mathbb{C}\right\}
$$

Let us address the question of linear, time-invariant (LTI) models for $\mathcal{D}$. We call the matrix $N(\xi)$ a representation of a model for $\mathfrak{B}$ in the generative sense if

1) $\operatorname{Im} N\left(\frac{d}{d t}\right) \exp _{\lambda_{i} t}=\mathcal{V}_{i}, i=1, \ldots n$.

2) $\operatorname{Im} N\left(\frac{d}{d t}\right) \exp _{\mu t}=\mathbb{C}^{\bullet}$ if $\mu \neq \lambda_{i}$ 


\section{B. Quadratic Differential Forms}

In systems theory, quadratic functionals of variables and their derivatives are often encountered, for instance, the Lyapunov function in stability theory, the Lagrangian of a dynamical system, the Hamiltonian, various cost criteria in optimal control, etc. In [8] a two variable polynomial matrix was used to represent such quadratic functionals. This notation has proved to be extremely handy. With this notation, operations on (infinite dimensional) trajectories can be conveniently represented as algebraic operations on (finite dimensional) polynomial matrices, albeit in two variables.

Let $\mathbb{R}^{q \times q}[\zeta, \eta]$ denote the set of real polynomial matrices in the indeterminates $\zeta$ and $\eta$. An element $\Phi$ in this set is given by

$$
\Phi(\zeta, \eta)=\sum_{k, l} \Phi_{k l} \zeta^{k} \eta^{l}
$$

where the sum ranges over non-negative integers $k, l$ and this sum is assumed finite (i.e. only a finite number of $\Phi_{k l}$ are nonzero). Such a $\Phi$ induces a quadratic differential form

$$
Q_{\Phi}: \mathcal{C}^{\infty}\left(\mathbb{R}, \mathbb{R}^{q}\right) \rightarrow \mathcal{C}^{\infty}(\mathbb{R}, \mathbb{R})
$$

defined by

$$
\left(Q_{\Phi}(w)\right)(t)=\sum_{k, l}\left(\frac{d^{k} w(t)}{d t^{k}}\right)^{T} \Phi_{k l}\left(\frac{d^{l} w(t)}{d t^{l}}\right) .
$$

Here, $\mathcal{C}^{\infty}\left(\mathbb{R}, \mathbb{R}^{\bullet}\right)$ denotes the space of $\mathcal{C}^{\infty}$ functions from $\mathbb{R}$ to $\mathbb{R}^{\bullet}$. $\Phi$ will be called symmetric if $\Phi(\zeta, \eta)=\Phi^{T}(\eta, \zeta)$. The set of such symmetric two variable polynomial matrices will be denoted by $\mathbb{R}_{s}^{q \times q}[\zeta, \eta]$. It can be seen that this set is actually a sub-ring of $\mathbb{R}^{q \times q}[\zeta, \eta]$. Moreover, it can be easily shown that it is enough to consider just symmetric two variable polynomial matrices and hence in this paper it will always be assumed that $\Phi \in \mathbb{R}_{s}^{q \times q}[\zeta, \eta]$.

A controllable linear differential behavior $\mathfrak{B} \equiv\{w\}$ is said to be dissipative with respect to the supply rate $Q_{\Phi}$, or in short $\Phi$-dissipative, if

$$
\int_{-\infty}^{\infty} Q_{\Phi}(w) d t \geq 0 \quad \forall \text { compactly supported } w \in \mathfrak{B}
$$

The set of all $\Phi$-dissipative controllable behaviors is denoted by $\mathcal{L}_{\Phi}$. There is a simple test to determine whether $\mathfrak{B}$ is $\Phi$ dissipative [8]: using an image representation $M\left(\frac{d}{d t}\right)$ of $\mathfrak{B}$, define the two variable polynomial matrix $\Phi^{\prime}(\zeta, \eta)$ by

$$
\Phi^{\prime}(\zeta, \eta)=M^{T}(\zeta) \Phi(\zeta, \eta) M(\eta)
$$

Proposition 2.1: $\mathfrak{B} \in \mathcal{L}_{\Phi}$ if and only if $\Phi^{\prime}(-i \omega, i \omega) \geq 0$ for all $\omega \in \mathbb{R}$.

A $\Phi$-dissipative behavior also has special associated QDFs known as the "storage functions". Every storage function, $Q_{\Psi}$, satisfies the "dissipation inequality":

$$
\frac{d}{d t} Q_{\Psi}(w) \leq Q_{\Phi}(w) \quad \forall w \in \mathfrak{B}
$$

We say that $Q_{\Psi}$ is a positive semidefinite storage function on the manifest variables of $\mathfrak{B}$ if for every manifest variable trajectory $w \in \mathfrak{B},\left(Q_{\Psi}(w)\right)(t) \geq 0$ for all $t \in \mathbb{R} . Q_{\Psi}$ is said to be a positive definite storage function on the manifest variables of $\mathfrak{B}$ if it is positive semidefinite, and in addition, $Q_{\Psi}(w)=0$ (i.e. $Q_{\Psi}(w)$ is the zero trajectory) $\Longleftrightarrow w=0$.

In this paper, we henceforth concentrate on behaviors with two manifest variables only. Assume that a controllable $\mathcal{C}^{\infty}$ behavior $\mathfrak{B}$ is given by the kernel representation

$$
\left[\begin{array}{ll}
p\left(\frac{d}{d t}\right) & -q\left(\frac{d}{d t}\right)
\end{array}\right]\left[\begin{array}{l}
u \\
y
\end{array}\right]=0
$$

An image representation of $\mathfrak{B}$ is seen to be

$$
\left[\begin{array}{l}
u \\
y
\end{array}\right]=\left[\begin{array}{l}
q\left(\frac{d}{d t}\right) \\
p\left(\frac{d}{d t}\right)
\end{array}\right] \ell
$$

Also, $\mathfrak{B}$ can be identified with the scalar rational function $p(\xi) / q(\xi)$. We shall invoke this association time and again in this paper. With this background, we are in a position to consider the NP interpolation problem in a behavioral setting, albeit for the simpler case of behaviors with two manifest variables. We develop the NP interpolation problem in two steps, first we develop it for the case when the interpolant satisfies a fixed norm condition at all frequencies. Later, we address the problem of an interpolant satisfying a frequency weighted norm condition.

\section{NEVANLINNA PICK INTERPOLATION - THE STANDARD CASE}

We begin by translating the classical Nevanlinna Pick interpolation problem as given in Section I into the language of behavioral systems theory and address some issues that arise out of such a formulation. We will only consider the scalar interpolation problem in this paper, and hence we consider $\mathcal{C}^{\infty}$-behaviors $\mathfrak{B}$ with two manifest variables $(u, y)$ that take values in $\mathbb{R}^{2}$. A behavioral formulation of Nevanlinna-Pick interpolation has been done in [7], where a characterization of solutions of a "Subspace Nevanlinna Interpolation Problem" was obtained in terms of kernel representations. In this paper we show obtain a characterization of all solutions in terms of image representations. Such a characterization has an advantage over [7]: controllability of all solutions obtained as image representations is guaranteed, unlike in a characterization in terms of kernel representations.

Consider a controllable behavior $\mathfrak{B}$ given by an observable image representation:

$$
\left[\begin{array}{l}
u \\
y
\end{array}\right]=\left[\begin{array}{l}
q\left(\frac{d}{d t}\right) \\
p\left(\frac{d}{d t}\right)
\end{array}\right] \ell
$$

Define the $2 \times 2$ constant matrix $J$ as

$$
J=\left[\begin{array}{cc}
1 & 0 \\
0 & -1
\end{array}\right]
$$

Let

$$
J_{\epsilon}^{\text {strict }}=\left[\begin{array}{cc}
1-\epsilon & 0 \\
0 & -1
\end{array}\right], \epsilon \in(0,1)
$$

Then, we have the following lemma:

Lemma 3.1: $\sup _{\omega \in \mathbb{R}}\left|\frac{p(i \omega)}{q(i \omega)}\right|<1$ if and only if the behavior $\mathfrak{B}$ associated with the rational function $p(\xi) / q(\xi)$ is $J_{\epsilon}^{\text {strict }}$-dissipative for some $\epsilon \in(0,1)$. 
Proof: Follows easily after applying Proposition 5.1 [8].

Lemma 3.1 serves as a connection between the classical (rational function based) formulation and a behavioral formulation of the NP problem.

Behavioral Nevanlinna Pick Interpolation (Problem statement): Consider $n$ trajectories $\left\{\mathcal{V}_{i} \exp _{\lambda_{i} t}\right\} i=1 \ldots n$ and $\exp _{x} \equiv e^{x}$, in $\mathcal{C}^{\infty}\left(\mathbb{R}, \mathbb{C}^{2}\right)$ (which we name the data set $\mathcal{D}$ ). Assume that

1) $\lambda_{i}, i=1,2 \ldots n \in \mathbb{C}^{+}$and are distinct,

2) $\mathcal{V}_{i} \in \mathbb{C}^{2}$ are contractive, i.e. $\mathcal{V}_{i}=\left[\begin{array}{ll}x y \\ ]^{T}\end{array}\right.$ $|y|$. Then,

determine all $J_{\epsilon}^{\text {strict }}$-dissipative controllable behaviors (i.e. all behaviors that are $J_{\epsilon}^{\text {strict }}$-dissipative for some $\epsilon \in(0,1)$ ) $\mathfrak{B}$ given by an observable image representation:

$$
\left[\begin{array}{l}
u \\
y
\end{array}\right]=\left[\begin{array}{l}
q\left(\frac{d}{d t}\right) \\
p\left(\frac{d}{d t}\right)
\end{array}\right] \ell
$$

such that:

1) $\mathfrak{B}$ has positive definite storage functions; 2) $\mathfrak{B} \quad$ contains $\quad \mathcal{D}, \quad$ i.e. $\left[\begin{array}{l}q\left(\frac{d}{d t}\right) \\ p\left(\frac{d}{d t}\right)\end{array}\right] \exp _{\lambda_{i} t}=$
$\mathcal{V}_{i} \exp _{\lambda_{i} t},, i=1,2 \ldots n$.

It is evident that condition (2) above implies that $\left[\begin{array}{l}q\left(\lambda_{i}\right) \\ p\left(\lambda_{i}\right)\end{array}\right] \exp _{\lambda_{i} t}=V_{i}, i=1,2 \ldots n$, where $V_{i}$ is a basis for $\mathcal{V}_{i}$. Thus, the problem is actually that of interpolating $n$ distinct subspaces $\mathcal{V}_{i} \exp _{\lambda_{i} t}, i=1, \ldots n$. We say that $\mathfrak{B}$ "interpolates the data" if it contains the trajectories $\mathcal{V}_{i} \exp _{\lambda_{i} t}$. Any behavior $\mathfrak{B}$ that satisfies conditions (1) and (2) will be called a solution to the "Subspace Nevanlinna Pick Problem (SNIP)".

We denote by $\mathcal{S}$ the linear span of the subspaces $\mathcal{V}_{i} \exp _{\lambda_{i} t}, i=1, \ldots n$. Thus $\mathcal{S}$ is a $\mathrm{n}$-dimensional subspace of $\mathcal{C}^{\infty}\left(\mathbb{R}, \mathbb{C}^{2}\right)$. We now introduce the concept of a "Pick matrix"of of the QDF $Q_{J}$ and the $n$ distinct subspaces $\mathcal{V}_{i} \exp _{\lambda_{i} t}, i=1,2 \ldots n$. A Pick matrix of $Q_{J}$ and $\mathcal{V}_{i} \exp _{\lambda_{i} t}$ is defined as the $n \times n$ Hermitian matrix:

$$
T_{J, \mathcal{V}_{1: n}, \lambda_{1: n}}=\left[\frac{V_{i}^{*} J V_{j}}{\overline{\bar{\lambda}}_{i}+\lambda_{j}}\right]_{i, j=1}^{n}
$$

where $V_{i} \in \mathbb{C}^{2}$ spans $\mathcal{V}_{i}$. A Pick matrix is obviously not unique, since a different basis for $\mathcal{V}_{i}$ gives a different Pick matrix. However it can be shown that properties of $T_{J, \mathcal{V}_{1: n}, \lambda_{1: n}}$ like its signature, sign-definiteness and (non)singularity remain invariant under a change of basis of the subspaces $\mathcal{V}_{i}$. While describing these properties, we shall refer to $T_{J, \mathcal{V}_{1: n}, \lambda_{1: n}}$ as the Pick matrix with a slight abuse of notation.

We now introduce the notion of the "dual" set of the data. Dualization is a very powerful concept that makes the NP problem simpler to solve (we address this in a later section).

\section{A. Dualizing of the data}

Given subspaces $\mathcal{V}_{i} \exp _{\lambda_{i} t}, i=1 \ldots n$ with $\mathcal{V}_{i} \in \mathbb{C}^{2}$ and contractive, define a "data set" $\mathcal{D}$ as

$$
\mathcal{D}=\left\{\mathcal{V}_{1} \exp _{\lambda_{1} t}, \mathcal{V}_{2} \exp _{\lambda_{2} t}, \ldots \mathcal{V}_{n} \exp _{\lambda_{n} t}\right\}
$$

Define the "dual subspace" of $\mathcal{V}_{i} \exp _{\lambda_{i} t}$ as $\mathcal{V}_{i}^{\perp_{J}} \exp _{-\bar{\lambda}_{i} t}$, where $\mathcal{V}_{i}^{\perp_{J}}$ is defined by:

$$
\mathcal{V}_{i}^{\perp_{J}}=\left\{v \in \mathbb{C}^{2} \mid v^{*} J w=0 \forall w \in \mathcal{V}_{i}\right\}
$$

Since $\mathcal{V}_{i}$ is contractive, $\mathcal{V}_{i}^{\perp_{J}}$ is uniquely defined and in fact the two are complements of one another in $\mathbb{C}^{2}$. The dual the data set $\mathcal{D}$ is defined by $\mathcal{D}^{\perp_{J}}$ in the obvious way:

$$
\mathcal{D}^{\perp_{J}}=\left\{\mathcal{V}_{1}^{\perp_{J}} \exp _{-\bar{\lambda}_{1} t}, \mathcal{V}_{2}^{\perp_{J}} \exp _{-\bar{\lambda}_{2} t}, \ldots \mathcal{V}_{n}^{\perp_{J}} \exp _{\bar{\lambda}_{n} t}\right\}
$$

Dualizing of the subspaces $\mathcal{V}_{i} \exp _{\lambda_{i} t}$ is instrumental in determining a characterization of all solutions to the SNIP, the following section shows.

\section{Construction of Nevanlinna-Pick INTERPOLANTS}

In the sequel we will assume that $T_{J, \mathcal{V}_{1: n}, \lambda_{1: n}}$ is a nonsingular (Hermitian) matrix. Then:

Theorem 4.1: If $T_{J, \mathcal{V}_{1: n}, \lambda_{1: n}}$ is nonsingular there exists a representation $N\left(\frac{d}{d t}\right)$ of a model for $\mathcal{D} \cup \mathcal{D}^{\perp_{J}}$ (in a generative sense) that satisfies the following properties:

1) $\operatorname{Im} N\left(\lambda_{i}\right)=\mathcal{V}_{i}$

2) $\operatorname{Im} N\left(-\bar{\lambda}_{i}\right)=\mathcal{V}_{i}^{\perp_{J}}$

3) $\operatorname{Im} N(\mu)=\mathbb{C}^{2}$ if $\mu \neq \lambda_{i},-\bar{\lambda}_{i}$

4) $N^{T}(-\xi) J N(\xi)=r(-\xi) r(\xi) J$

for some polynomial $r(\xi) \in \mathbb{R}[\xi]$.

Proof: We only give a short proof of the result due to limitations of space. Consider the trajectory $\mathcal{V}_{1} \exp _{\lambda_{1} t}$. Define a matrix $R(\xi)$ as follows:

$$
R(\xi)=\left(\xi+\bar{\lambda}_{1}\right) I^{2 \times 2}-V_{1}\left[\frac{V_{1}^{*} J V_{1}}{\lambda_{1}+\bar{\lambda}_{1}}\right]^{-1} V_{1}^{*} J
$$

Then, it can be shown that

1) $R\left(\frac{d}{d t}\right) \mathcal{V}_{1} \exp _{\lambda_{1} t}=0$

2) $R\left(\frac{d}{d t}\right) \mathcal{V}_{1}^{\perp J} \exp _{-\bar{\lambda}_{1} t}=0$.

3) $R^{T}(-\xi) J R(\xi)=\left(-\xi-\bar{\lambda}_{1}\right)\left(\xi+\lambda_{1}\right) J$.

Define $N(\xi)=\operatorname{adj} R(\xi)$, i.e. $R(\xi) N(\xi)=\operatorname{det} R(\xi) I^{2 \times 2}$. Since $\operatorname{det} R(\xi)$ has roots at $\lambda_{1}$ and $-\bar{\lambda}_{1}, R\left(\lambda_{1}\right) N\left(\lambda_{1}\right)=0$ and $R\left(-\bar{\lambda}_{1}\right) N\left(-\overline{\lambda_{1}}\right)=0$. Thus, the differential operator $N\left(\frac{d}{d t}\right)$ satisfies the properties:

1) $\operatorname{Im} N\left(\frac{d}{d t}\right) \exp _{\lambda_{1} t}=\mathcal{V}_{i}$ and

2) $\operatorname{Im} N\left(\frac{d}{d t}\right) \exp _{-\overline{\lambda_{1} t}}=\mathcal{V}_{1}^{\perp_{J}}$.

It can be easily verified that $N^{T}(-\xi) J N(\xi)=$ $\left(\xi+\lambda_{1}\right)\left(-\xi+\bar{\lambda}_{1}\right) J$. A recursive modeling procedure developed in [6] can be used to construct $N\left(\frac{d}{d t}\right)$ for $\mathcal{V}_{2} \exp _{\lambda_{2} t} \ldots \mathcal{V}_{n} \exp _{\lambda_{n} t}$ and their duals.

This "special" representation of a MPUM for $\mathcal{D} \cup \mathcal{D}^{\perp_{J}}$ can be used to elegantly characterize all solutions of the SNIP: 
Theorem 4.2: Consider the data set $\mathcal{D} \cup \mathcal{D}^{\perp_{J}}$. Let $N(\xi)$ be a "special representation" of a MPUM for $\mathcal{D} \cup \mathcal{D}^{\perp_{J}}$ as defined in Theorem 4.1. The following statements are equivalent:

1) The Pick matrix $T_{J, \mathcal{V}_{1: n}, \lambda_{1: n}}$ is positive definite.

2) There exists a solution to the SNIP.

Further, every solution $\operatorname{Im}\left[\begin{array}{c}q\left(\frac{d}{d t}\right) \\ p\left(\frac{d}{d t}\right)\end{array}\right]$ to the SNIP can be given as

$$
f(\xi)\left[\begin{array}{c}
q(\xi) \\
p(\xi)
\end{array}\right]=N(\xi)\left[\begin{array}{l}
\phi(\xi) \\
\pi(\xi)
\end{array}\right]
$$

where $f(\xi)$ is a Hurwitz polynomial and $\|\pi(\xi) / \phi(\xi)\|_{\infty}<1$.

Proof: We give a sketch of the complete proof. Since $N(\xi)$ is $J$-unitary, every $J$-dissipative behavior is dissipative with respect to the QDF induced by $N^{T}(\zeta) J N(\eta)$ and vice versa. Hence, every solution to the interpolation problem is obtained as the image of a $J$-dissipative behavior under the map $N\left(\frac{d}{d t}\right)$. A $J$-dissipative behavior defined by $\mathrm{Im}$ $\left[\begin{array}{l}q\left(\frac{d}{d t}\right) \\ p\left(\frac{d}{d t}\right)\end{array}\right]$ has positive definite storage functions if and only if $q(\xi)$ is Hurwitz. It can be shown that if $\phi(\xi)$ (in the Theorem statement) is chosen to be Hurwitz, $q(\xi)$ is guaranteed to be Hurwitz. Hence, every solution to the SNIP can be obtained as the image of the set of all plants having infinity norm less than one, under the map $N\left(\frac{d}{d t}\right)$.

An important advantage of converting the SNIP from a kernel representation to an image representation is that by doing so, controllability is guaranteed. Such a re-formulation also explains why dualizing the data is crucial in solving the Nevanlinna-Pick interpolation problem.

Notice that in the solution suggested above, we have considered the data set $\mathcal{D} \cup \mathcal{D}^{\perp_{J}}$. This is in contrast with the problem statement of SNIP which related to finding all interpolating behaviors for $\mathcal{D}$ alone. The following section addresses the necessity of considering $\mathcal{D} \cup \mathcal{D}^{\perp_{J}}$ rather than $\mathcal{D}$ alone, and how this consideration still yields all solutions to SNIP.

\section{SYSTEM THEORETIC IMPLICATIONS OF DUALIZING THE DATA}

In this section, we give a justification for dualizing the data. We start by considering a hypothetical situation in which the data has not been dualized. Let as before $\mathcal{D}=$ $\left\{\mathcal{V}_{i} \exp _{\lambda_{i} t}\right\}$ be the data set and $F(\xi)$ be any model in the generative sense for $\mathcal{D}$, i.e. $\operatorname{Im} F\left(\lambda_{i}\right)=\mathcal{V}_{i}$ and $\operatorname{Im}$ $F(\mu)=\mathbb{C}^{2}, \mu \neq \lambda_{i}$. Then, the following lemma is easily proved:

Lemma 5.1: Given any representation of a model $F\left(\frac{d}{d t}\right)$ (in the generative sense) of $\mathcal{D}$, let

$$
\left[\begin{array}{l}
q(\xi) \\
p(\xi)
\end{array}\right]=F(\xi)\left[\begin{array}{l}
s(\xi) \\
r(\xi)
\end{array}\right]
$$

Then, $\operatorname{Im}\left[\begin{array}{c}q\left(\lambda_{i}\right) \\ p\left(\lambda_{i}\right)\end{array}\right] \subseteq \mathcal{V}_{i}$ for arbitrary $s(\xi), r(\xi)$.

Proof: Follows from the fact that $\operatorname{Im} F\left(\lambda_{i}\right)=\mathcal{V}_{i}$.
Corollary 5.2: Every behavior $\mathfrak{B}=\operatorname{Im}\left[\begin{array}{c}q\left(\frac{d}{d t}\right) \\ p\left(\frac{d}{d t}\right)\end{array}\right]$ which interpolates given subspaces $\mathcal{V}_{i} \exp _{\lambda_{i} t}$ is given as $\left[\begin{array}{l}q(\xi) \\ p(\xi)\end{array}\right]=F(\xi)\left[\begin{array}{l}s(\xi) \\ r(\xi)\end{array}\right]$ where $s(\xi), r(\xi)$ are arbitrary coprime polynomials.

At this juncture when all possible interpolants have been characterized, we bring in the additional condition of dissipativity. Consider the two variable polynomial matrix $\Phi(\zeta, \eta)$ defined as

$$
\Phi(\zeta, \eta)=F^{T}(\zeta) J F(\eta)
$$

The following theorem relates $\Phi$-dissipativity with solutions to SNIP:

Theorem 5.3: Consider the set of $\Phi$-dissipative behaviors. Let $\mathfrak{B}^{\prime}$ be an arbitrary behavior in this set. Then, $\mathfrak{B}:=$ $F\left(\frac{d}{d t}\right)\left(\mathfrak{B}^{\prime}\right)$ is a $J$-dissipative behavior that interpolates $\mathcal{D}:=$ $\mathcal{V}_{i} \exp _{\lambda_{i} t}$. Moreover, for every $J$-dissipative behavior $\mathfrak{B}$ that interpolates $\mathcal{D}$ there exists a corresponding $\Phi$-dissipative behavior $\mathfrak{B}^{\prime}$.

Proof: It has been shown in [3] that $F\left(\frac{d}{d t}\right)$ can be thought of as a differential operator that maps every $\Phi$-dissipative behavior into a $J$-dissipative behavior. Moreover, it is "invertible", i.e. for every $J$-dissipative behavior, there exists a corresponding $\Phi$-dissipative behavior and vice versa.

A behavior $\mathfrak{B}$ that is given by $\operatorname{Im} F\left(\frac{d}{d t}\right)$ interpolates $\mathcal{D}$ since $F(\xi)$ is a model for $\mathcal{D}$. Further, such a $\mathfrak{B}$ is $J$-dissipative if and only if it is obtained as the image of a $\Phi$-dissipative behavior under the map $F\left(\frac{d}{d t}\right)$.

Thus in principle given any representation of a model for $\mathcal{D}$, one may construct a QDF $Q_{\Phi}$ from this representation. If the set of $\Phi$-dissipative behaviors is "known", the set of all $J$-dissipative behaviors that interpolate $\mathcal{D}$ can be determined.

One sees immediately that $Q_{\Phi}$ is a fairly general supply rate and no easy characterization is available for the set of $\Phi$-dissipative behaviors. Of course, we do have a characterization in terms of $J$-dissipative behaviors [3] but this characterization is not good enough in the present context. Thus, determining the set of $\Phi$-dissipative behaviors is arguably a difficult task in general. We try to make $Q_{\Phi}$ "as simple as possible" so that the set of $\Phi$-dissipative behaviors is "known". Dualizing the data does just this, as we show below.

Assume that the model $F(\xi)$, in addition to modeling $\mathcal{D}$ also models $\mathcal{D}^{\perp_{J}}$ (in a generative sense). Then we have the following result:

Theorem 5.4: The representation $F(\xi)$, which is a representation of a model for $\mathcal{D} \cup \mathcal{D}^{\perp_{J}}$, satisfies

$$
F^{T}(-\xi) J F(\xi)=r(\xi) r(-\xi) U^{T}(-\xi) J U(\xi)
$$

with $U(\xi)$ unimodular if and only if columns of $F\left(-\bar{\lambda}_{i}\right)$ are $J$-orthogonal to columns of $F\left(\lambda_{i}\right)$, i.e. $F^{T}\left(-\bar{\lambda}_{i}\right) J F\left(\lambda_{i}\right)=0$

Proof: Assume $F^{T}\left(-\bar{\lambda}_{i}\right) J F\left(\lambda_{i}\right)=0$. Since $F(\xi)$ is nonsingular, $F^{T}(-\xi) J F(\xi)$ is a nonzero polynomial. Hence, 
there exists a polynomial $r(\xi)$ such that $r(\xi) r(-\xi)$ divides $F^{T}(-\xi) J F(\xi)$. Notice that the inertia of $F^{T}(-\xi) J F(\xi)$ is the same as that of $J$ for all $\xi \neq \lambda_{i},-\bar{\lambda}_{i}$. Also, since $\operatorname{det} F^{T}(-\xi) J F(\xi)$ has no other roots than $\lambda_{i},-\bar{\lambda}_{i}$, there exists a Unimodular matrix $U(\xi)$ such that $F^{T}(-\xi) J F(\xi)=$ $r(\xi) r(-\xi) U^{T}(-\xi) J U(\xi)$.

The converse of the statement is trivial.

Remark 5.5: The surprising, and interesting feature of the Nevanlinna-Pick iteration algorithm is that there exists a representation of a model with $U(\xi)$ (see Theorem 5.4) equal to the identity matrix. Indeed, if $F(\xi)$ is a model in the generative sense, $F(\xi) V(\xi)$ is also another representation of the same model if and only if $V(\xi)$ is unimodular. This follows from the fact that the columns of $F(\xi)$ and that of $F(\xi) V(\xi)$ generate the same module. Thus, given any matrix $F(\xi)$ such that columns of $F\left(\lambda_{i}\right)$ are $J$-orthogonal to columns of $F\left(-\bar{\lambda}_{i}\right)$ there exists a unimodular matrix $V(\xi)$ such that $F_{1}(\xi)=F(\xi) V(\xi)$ satisfies $F_{1}^{T}(-\xi) J F_{1}(\xi)=$ $r(\xi) r(-\xi) J$.

In other words, Theorem 5.4 and Remark 5.5 imply that if $\operatorname{Im} F\left(-\bar{\lambda}_{i}\right)$ models $\mathcal{D}^{\perp_{J}}$ then $F(\xi)$ can be suitably modified so that it enjoys the $J$-unitary property. In the following subsection, another implication of dualizing is addressed with reference to Pick matrices.

\section{A. Dualizing and Pick matrices}

Given a $J$-dissipative behavior $\mathfrak{B}$ with two manifest variables given by $\mathfrak{B}=\operatorname{Im}\left[\begin{array}{c}q\left(\frac{d}{d t}\right) \\ p\left(\frac{d}{d t}\right)\end{array}\right]$, the Pick-matrix $T$ associated with $\mathfrak{B}$ is defined as follows:

1) Compute roots of $\lambda_{i}$ of $q(-\xi) q(\xi)-p(-\xi) p(\xi)=0$ such that $\lambda_{i} \in \mathbb{C}^{+}, i=1,2 \ldots n$.

2) Then $T=\left[\frac{q\left(\bar{\lambda}_{i}\right) q\left(\lambda_{j}\right)-p\left(\bar{\lambda}_{j}\right) p\left(\lambda_{i}\right)}{\lambda_{j}+\bar{\lambda}_{i}}\right]_{i, j=1}^{n}$ is the Pick matrix.

In SNIP, a necessary and sufficient condition for solvability is stated in terms of a Pick matrix. This matrix is completely dependent on the data given and has (apparently) no connection with the Pick matrix of an interpolating behavior. However, we now show that because of dualizing the data, these two objects are in fact the same (modulo a congruence transformation).

Given the data set $\mathcal{D}=\mathcal{V}_{i} \exp _{\lambda_{i} t}$ with $\lambda_{i} \in \mathbb{C}^{+}$, recall that the Pick matrix of the data is defined as

$$
T_{J, \mathcal{V}_{1: n}, \lambda_{1: n}}=\left[\frac{V_{i}^{*} J V_{j}}{\overline{\lambda_{i}}+\lambda_{j}}\right]_{i, j=1}^{n}
$$

Consider a behavior $\mathfrak{B}$ associated with the rational function $p(\xi) / q(\xi)$ that is a solution to the SNIP. Therefore, it follows that

$$
\operatorname{Im}\left[\begin{array}{c}
q\left(\lambda_{i}\right) \\
p\left(\lambda_{i}\right)
\end{array}\right]=\mathcal{V}_{i}
$$

Since the "special model"(Theorem 4.1 ) models $\mathcal{D} \cup$ $\mathcal{D}^{\perp_{J}}$, any interpolant models both $\mathcal{D}$ and $\mathcal{D}^{\perp_{J}}$. This is in apparently more restrictive than the problem statement which required $\mathfrak{B}$ to interpolate only $\mathcal{D}$. However, the characterization obtained in Theorem 4.2 shows that a solution to the SNIP may be obtained with a common factor that is a Hurwitz polynomial, in other words a polynomial having roots at $-\bar{\lambda}_{i}$. Thus, $\mathfrak{B}$ does interpolate $\mathcal{D}^{\perp J}$, albeit in a trivial manner.

Thus, in summary, dualizing the data has the following system theoretic implications:

- It is necessary for, and guarantees existence of a $J$ unitary model $F\left(\frac{d}{d t}\right)$.

- $J$-unitariness of a model implies that the QDF $Q_{\Phi}$ defined by $\Phi(\zeta, \eta)=F^{T}(\zeta) J F(\eta)$ is "like $Q_{J}$ ", i.e. every $\Phi$-dissipative behavior is $J$-dissipative and vice versa. Thus, the set of $\Phi$-dissipative behaviors in this case is "known" which enables a easy characterization of the solutions of the SNIP.

We now address a generalization of SNIP using Quadratic differential forms. We obtain a characterization of interpolants that satisfy a frequency-dependent norm, a result which is new and important.

\section{NEVANLINNA-PICK PROBLEM WITH FREQUENCY DEPENDENT NORMS}

In this section, we address the problem of constructing $\Phi$ dissipative behaviors that interpolate certain given subspaces. The matrix $\Phi$ that induces the QDF $Q_{\Phi}$ may not necessarily be a constant matrix. Hence, interpolating behaviors that are $\Phi$-dissipative are required to satisfy a "frequency dependent norm" along with the given interpolation conditions.

We assume that the $\mathrm{QDF} Q_{\Phi}$ is such that $\Phi(\zeta, \eta)$ admits the factorization

$$
\Phi(\zeta, \eta)=K^{T}(\zeta) J_{\epsilon}^{\text {strict }} K(\eta)
$$

with $K(\xi)$ square and nonsingular. Necessary and sufficient conditions for such a factorization (and an algorithm to compute the factorization when it exists) can be easily obtained using the coefficient matrix of a QDF. We now state the "generalized SNIP" (abbreviated as GSNIP) which we will address in this section:

GSNIP: Given a QDF $Q_{\Phi}$ with $\Phi(\zeta, \eta)=$ $K^{T}(\zeta) J_{\epsilon}^{\text {strict }} K(\eta)$ with $K(\xi) \in \mathbb{R}^{2 \times 2}[\xi]$ and nonsingular, together with $n$ distinct subspaces $\mathcal{V}_{i} \exp _{\lambda_{i} t}$, determine necessary and sufficient conditions for the existence of $\Phi$-dissipative behaviors $\mathfrak{B}:=\operatorname{Im}\left[\begin{array}{l}q\left(\frac{d}{d t}\right) \\ p\left(\frac{d}{d t}\right)\end{array}\right]$ such that

1) $\mathfrak{B}$ has positive definite storage functions (with respect to $\left.Q_{\Phi}\right)$, and

2) $\operatorname{Im}\left[\begin{array}{l}q\left(\lambda_{i}\right) \\ p\left(\lambda_{i}\right)\end{array}\right]=\mathcal{V}_{i}$

Assumption: We assume that $\left\{\lambda_{i}\right\} \cap \operatorname{spec} K(\xi)=\phi$, i.e. $\lambda_{i}$ is not a singularity of $K(\xi)$. We also assume that the spaces $K\left(\lambda_{i}\right) \mathcal{V}_{i}$ are contractive.

The following theorem gives necessary and sufficient conditions for the solvability of GSNIP:

Theorem 6.1: Given

1) a $\mathrm{QDF} Q_{\Phi}$ with $\Phi(\zeta, \eta)=K^{T}(\zeta) J_{\epsilon}^{\text {strict }} K(\eta)$. 
2) a $\Phi$-dissipative $\mathcal{C}^{\infty}$-behavior $\mathfrak{B}$ defined by an observable image representation:

$$
\left[\begin{array}{l}
u \\
y
\end{array}\right]=\left[\begin{array}{l}
q\left(\frac{d}{d t}\right) \\
p\left(\frac{d}{d t}\right)
\end{array}\right] \ell
$$

3) subspaces $\mathcal{V}_{i} \exp _{\lambda_{i} t}$ with $\mathcal{V}_{i} \in \mathbb{C}^{2}, \lambda_{i} \in \mathbb{C}^{+}$ the GSNIP is solvable if and only if

1) $\left[\begin{array}{c}s(\xi) \\ r(\xi)\end{array}\right]=K(\xi)\left[\begin{array}{c}q(\xi) \\ p(\xi)\end{array}\right]$ are such that $r(\xi), s(\xi)$

2) The modified pick matrix $T_{\Phi, \mathcal{V}_{1: n}, \lambda_{1: n}}$ is positive definite where

$$
T_{\Phi, \mathcal{V}_{1: n}, \lambda_{1: n}}=\left[\frac{V_{i}^{*} K^{T}\left(\bar{\lambda}_{j}\right) J K\left(\lambda_{i}\right) V_{i}}{\lambda_{i}+\bar{\lambda}_{j}}\right]_{i, j=1}^{n}
$$

Further, every $\Phi$-dissipative behavior $\mathfrak{B}$ with positive definite storage functions that interpolates $\mathcal{V}_{i} \exp _{\lambda_{i} t}$ satisfies conditions 1 and 2.

Proof: We give a sketch of the complete proof in the following lines. Define a behavior $\mathfrak{B}^{\prime}$ as $\operatorname{Im}\left[\begin{array}{c}s\left(\frac{d}{d t}\right) \\ r\left(\frac{d}{d t}\right)\end{array}\right] \ell$. Since $\mathfrak{B}$ is $\Phi$-dissipative, $\mathfrak{B}^{\prime}$ is $J_{\epsilon}^{\text {strict }}$-dissipative.

The behavior $\mathfrak{B}$ has positive definite storage functions if and only if $r(\xi)$ and $s(\xi)$ are coprime, and $s(\xi)$ is Hurwitz. This follows from the Kalman-Yakubovich lemma [4].

Define $W_{i}=K\left(\lambda_{i}\right) V_{i}$. Then, there exists a $\Phi$-dissipative interpolating behavior $\mathfrak{B}$ for $\mathcal{V}_{i} \exp _{\lambda_{i} t}$ if and only if there exists a $J_{\epsilon}^{\text {strict }}$-dissipative behavior $\mathfrak{B}^{\prime}$ for $W_{i} \exp _{\lambda_{i} t}$.

Finally, there exists a solution to the SNIP (a "stable" $\mathfrak{B}^{\prime}$ ) if and only if the corresponding Pick matrix is positive definite:

$$
T_{J, \mathcal{V}_{1: n}, \lambda_{1: n}}=\left[\frac{W_{i}^{*} J W_{j}}{\bar{\lambda}_{i}+\lambda_{j}}\right]_{i, j=1}^{n}>0
$$

This argument shows that there exists a $\Phi$-dissipative behavior that interpolates $\mathcal{V}_{i} \exp _{\lambda_{i} t}$ if and only if the coprimeness conditions hold, and if in addition the modified Pick matrix is positive definite. The fact that every such solution must be obtained using the procedure suggested follows from a simple contradiction argument.

The essential idea in the above proof is that the matrix $K(\xi)$ can be used to convert the problem into a problem of SNIP with $Q_{J_{\epsilon}^{\text {strict }}}$. Thus solution to GSNIP can be obtained as follows:

1) Given subspaces $\mathcal{V}_{i} \exp _{\lambda_{i} t}$ choose a basis $V_{i}$ for $\mathcal{V}_{i}$ Define

$$
W_{i}=K\left(\lambda_{i}\right) V_{i}
$$

2) Compute the Pick matrix $\left[W_{i}^{*} J W_{j} /\left(\bar{\lambda}_{i}+\lambda_{j}\right)\right]_{i, j=1}^{n}$. If this matrix is positive definite then proceed, else stop, there is no solution.

3) Compute all $J_{\epsilon}^{\text {strict }}$-dissipative behaviors that interpolate the subspaces $W_{i} \exp _{\lambda_{i} t}$, and which have positive definite storage functions. Let $\mathfrak{B}^{\prime}$ be such a (controllable) behavior.

4) Every behavior $\mathfrak{B}$ that satisfies the condition that there exists a $\mathfrak{B}^{\prime}$ (with an observable image representation) satisfying $\mathfrak{B}=K\left(\frac{d}{d t}\right)\left(\mathfrak{B}^{\prime}\right)$ is a solution to GSNIP.

\section{CONCLUSION}

In this paper we have provided a behavioral theoretic characterization of all solutions to the Nevanlinna Pick interpolation problem. The characterization presented here guarantees controllability. We have provided an explanation as to the need of the so called "mirror images" in interpolation problems. In all classical formulations of the NevanlinnaPick problems, the interpolant is required to satisfy a "frequency independent" norm condition. We have generalized the Nevanlinna-Pick problem to cases where a interpolant is required to satisfy a "frequency dependent" norm condition. This is shown to be intimately related to dissipativity with respect to a supply rate defined using a Quadratic Differential Form. We have obtained necessary and sufficient conditions for the solvability of a class of Nevanlinna-Pick interpolation problems with "frequency dependent" norm conditions.

\section{REFERENCES}

[1] A.C Antoulas, J.C. Willems, "A Behavioral Approach to Linear Exact Modeling", IEEE Transactions on Automatic Control, 38 (1993), pp 1776-1801.

[2] J.A. Ball, I. Gohberg, L. Rodman, Interpolation of Rational Matrix Functions, Birkhauser-Verlag, 1990.

[3] I. Pendharkar, H.K. Pillai, "A parametrization for dissipative behaviors", Systems and Control Letters, 51 (2004), pp 123-132.

[4] I. Pendharkar, H.K. Pillai, "Kalman-Yakubovich lemma in the behavioral setting", International Journal on Control, Accepted for publication.

[5] J.W. Polderman, J.C. Willems, "Introduction to mathematical systems theory: A behavioral approach", Springer-Verlag, 1997.

[6] P. Rapisarda, Linear Differential Systems, Ph.D. Thesis, University of Groningen, 1998.

[7] P. Rapisarda, J.C. Willems "The subspace Nevanlinna-Pick interpolation problem and the most powerful unfalsified model", Systems and control letters, 32(1997) pp 291-300.

[8] J.C. Willems, H.L. Trentelman, "On Quadratic Differential Forms", SIAM Journal of Control and Optimization, 36 (1998), pp 1703-1749. 